\title{
Knotworking in an emergency response team: understanding team communication and process
}

\author{
Janet W. Colvin \\ Communication Department, Utah Valley University, Orem, UT, USA
}

\begin{abstract}
Over the last 30 years, many studies have focused on responses to crisis in organizations. Crises can occur at any time of the day or night on a regular but unforeseen basis and are characterized by high consequences and short decision time. In healthcare, multidisciplinary crisis management or rapid response teams (RRT) have become more common. RRTs allow for a cross-sectional focus on patients' needs and, thereby, prevent avoidable deaths or significant harm. This study uses Activity Theory and knotworking theory to examine how communication, multiple roles and power structures are negotiated in RRT's in a large intermountain teaching hospital. Results from focus groups indicate a single-minded focus on training for system errors may miss important aspects. Knotworking theory is promising because it helps providers move beyond an understanding of teamwork to an understanding of how objectives can be shared and merged.
\end{abstract}

\section{Introduction}

Over the last 30 years, many studies have focused on how organizations respond to crisis. Crises can occur at any time on a regular but unforeseen basis, and are characterized by high consequences and short decision times. ${ }^{1}$ Stachowski and Kaplan ${ }^{2}$ suggest that understanding how teams carry out tasks, shed routine interaction patterns and

Correspondence: Janet W. Colvin, Communication Department, Utah Valley University, $800 \mathrm{~W}$. University Parkway, Orem, UT, USA.

Tel.: +1.801.863.7282 - Fax: +1.801 .863 .7287$

E-mail: colvinja@uvu.edu

Key words: crisis, teams, knotworking, activity theory.

Contributions: the authors contributed equally.

Conflict of interest: the authors declare no potential conflict of interest.

Contributions: JWC is the sole author on this paper. James Anderson, Nancy Tobler, Janet Fisher all contributed to the research project and indirectly to the paper.

Received for publication: 2 October 2017.

Revision received: 30 December 2127

Accepted for publication: 12 January 2018.

This work is licensed under a Creative Commons Attribution NonCommercial 4.0 License (CC BY-NC 4.0).

CCopyright J.W. Colvin et al., 2017

Licensee PAGEPress, Italy

Qualitative Research in Medicine \& Healthcare 2017; 1:128-137

doi:10.4081/qrmh.2017.7115 adapt to crisis situations across a variety of circumstances is an important area for study.

In healthcare, multidisciplinary crisis management or rapid response teams (RRT) have become more common, allowing a cross-sectional focus on patients' needs, thereby preventing death or significant harm to patients. Various participants may differ on how they believe communication occurs and should occur between team members, how and when team training should occur, and how teams should work together to intervene and prevent adverse outcomes.

The following study provides an analysis of an RRT to help further the understanding of the crucial team processes that may occur during crises in healthcare organizations. To understand RRTs processes in labor and delivery centers, prior research on RRTs is reviewed, and knotworking theory is explained and applied to a study of a labor and delivery center at a large teaching hospital.

\section{Rapid response teams}

In all teams, communication is important to assure the team meets their goal(s). Team processes such as the ability to work together and communicate effectively improve care and increase patients' understanding of procedures and feelings of satisfaction. When patients are experiencing distress, RRTs are called together as a team of diverse caregivers to prevent further deterioration and adverse outcomes $^{3}$ by managing patients' crises. RRTs have been examined in medical situations such as emergency rooms, ${ }^{4}$ intensive care, ${ }^{5}$ and cardiac care units across multiple hospitals and locations. ${ }^{6}$ Multiple studies have focused on how training impacts RRTs. In their meta-analysis of RRTs, Chan and colleagues ${ }^{7}$ note a heterogeneity across reported outcomes. They suggest this 
may be due to a lack of standardized terms and treatment protocols. In their study, Leach and $\mathrm{Mayo}^{3}$ note that most of the research on response teams has focused on tracking and reporting the hospital outcomes (p. 199) and much remains unknown about teams and their performance during crises because of the complexity involved.

Power imbalances between team members explains part of the complexity in RRTs. Team members have different levels of knowledge and expertise. They also have different levels of responsibility and power. Power may not be equally distributed and is dynamic is RRTs. ${ }^{8}$ For example, physicians may have formal power but nurses might have more experience especially at a teaching hospital. Not only is healthcare complex, the complexity is compounded by a tradition of professional fragmentation, of individualism, of a well-entrenched hierarchical authority structure, and of diffuse accountability ${ }^{9}$ (p. 576). In a meta-analysis of collaboration research in healthcare, San Martín-Rodríguez and colleagues (2005) identified social structures as a key influence on team behavior.

\section{Activity theory}

One of the main reasons why teamwork in RRTs remains elusive may be that tying together seemingly separate threads of activity ${ }^{10}$ with different power dynamics, team members, roles, personnel rotations, and ways of communicating is a complex and ever changing process. Engestrom says that any complex social encounter - like those involved in interprofessional teamwork and communication - should take into consideration entire activity systems where multiple roles, history, points of view, and interests all intersect. ${ }^{11}$ The concept of an activity system suggests that an activity is not a homogenous entity but rather disparate group of elements, voices and viewpoints. Kuutti ${ }^{12}$ notes that the purpose of such an activity system has its origin in a need and is geared towards producing a specific outcome. In this case the activity is the coming together of an emergency team in order to save a patient's life. Elements that contribute to an activity have been put into an activity system. ${ }^{13}$

An activity system is composed of six elements: individuals, objectives, tools, communities, rules, and division of labor. These elements demonstrate how interconnected a person's objective is to resources, social community, rules and structure, and division of labor. When different objectives become shared in a crisis situation, unless those objectives can be merged, deviations or contradictions may occur leading to breakdown in communication, errors, and even endangerment of the patient. Engestrom ${ }^{14}$ suggests that the more clearly we understand the discourse and its relationship to local productive activity, the more we will understand what is actually happening is teamwork.

\section{Knotworking}

While literature on interprofessional teamwork in health care abounds, little if any has examined the complicated activity and discourse that occurs as different stakeholders interact. Interaction occurs as actors help determine the actions of others. Actors in an RRT work together to enable patient care. As they interact, they use discourse which enables the activity to occur. Discourse reflects the historically constituted social/collective action of meaning construction in a community ${ }^{15}$ and relates to how participants' language serves to regulate interpersonal relations and in turn is produced through patterns of interpersonal relations. For example, in an RRT, a physician may use acronyms or specific medical terms which everyone understands. However, they may also issue abrupt commands to nurses and medical assistants which make them feel they are being talked down to or disrespected. In turn, feeling disrespected may create an atmosphere of distrust and toxicity as team members have to work together.

Activity theory supports the study of complex situations such as RRTs. Even more specific to health care is its subtheory of knotworking. Engestrom, Engestrom and Vahaaho, ${ }^{16}$ study threads of activity in complex multi-professionals settings that are rapidly changing and improvised as co-configuration and collaborative performance take place. When these practices go beyond conventional teamwork or networking to incorporate loosely connected actors and their work systems Engestrom et al., argue that such co-configuration moves to the practice of knotworking. Knotworking occurs when no single actor has fixed responsibilities or control but rather, when expertise is distributed across rapidly changing work places. Knotworking is characterized by a pulsating, distributed and partially improvised orchestration of collaborative performance between otherwise loosely connected actors and activity systems ${ }^{16}$ (p. 346).

In knotworking, because the crisis changes from moment to moment and involves constantly changing combinations of individuals distributed over time and space, the knot or center cannot be reduced to a particular individual or organizational center. The consistency over time is the ongoing mix of interaction among contributors, discourses, tasks and tools. The following is a hypothetical example of an RRT center:

Ms. Jones has been laboring for the past 12 hours. Suddenly the baby's heart rate drops to a dangerous level and is not coming back up despite interventions to help it improve. The labor \& delivery nurse is alone in the room with the patient and her husband and she knows she needs help.

She pushes the emergency call light and asks the unit secretary to initiate an $O B$ rapid response initiating a series of procedures that have been developed over time to deal with emergency situations. The unit secretary sends out the page and members of the response team are alerted to proceed to the patient's room to evaluate her and assist in this emergency. The obstetrician informs the patient that a cesarean section is indicated and the team is activated and quickly 
moves the patient to the operating room and prepares to proceed with the surgery.

The room is busy but all team members are very focused. The scene includes a nurse who is helping to move the patient to the OR table and position her correctly another nurse is prepping the patient's abdomen for surgery. The obstetricians are scrubbing their hands in preparation for the surgery. A scrub tech is setting up the instruments and is prepared to gown and glove the surgeons when they come in. The patient has a labor epidural and the anesthesiologist is checking her anesthesia level and giving more anesthetic for the cesarean. In addition to the $O B$ team, a pediatric resuscitation team is readying their equipment to be prepared to assist the baby when born if needed.

A knot could occur at any point of this activity. Because of lack of experience, the labor and delivery nurse checking on the patient may not call an emergency soon enough, the cart with supplies may be missing certain instruments, the staff nurse and the obstetrician may not get along and because of their personal differences do not communicate as clearly as they might otherwise, the anesthesiologist may not have heard their page and the rest of the team had to wait until she could arrive. Different interests, values, and practices brought by each member/role must be mediated in the knot. Engestrom ${ }^{17}$ argues that such mediation, in order to be successful, requires development of procedures and analyses to enable engagement in the knots and more frequent (re)negotiation than occurs in other types of organizational systems. Thus, procedures such as what constitutes an emergency, who performs which tasks, or what happens if someone on the team does not respond not only acknowledge that knots will occur but also what should happen to address those knots.

Each professional comes to the team from a unique activity system and contributes a unique role in providing patients' care. Each professional is dynamically changing and through a relatively short period of time, overlap of roles, without clear-cut boundaries, and distributed collaborative work process, create knots as they co-configure tasks and tools. Over time, the roles may stay the same; however, different individuals enact those roles as shifts change and different collaborators enter or leave the care of a patient.

Engestrom ${ }^{18}$ maintains that when an organization is trying to implement change that a linear process does not work as it ignores agency and motivational dynamics. Even as accounting for agency allows stakeholders to change and adapt, it also creates situations rife with the possibility of misunderstanding, uncertainty and resistance. Engestrom suggests five steps to allow for participants' agentic actions: i) Resisting the interventionist or management. ii) Explicating new possibilities or potential in the activity. iii) Envisioning new patterns or models of the activity. iv) Committing to concrete actions aimed at changing the activity and expressing this through commissive speech acts. v) Taking consequential actions to change the activity.
One way that agentic actions and motivational dynamics can be studied is through the examination of organizational learning and power in knots. Power gets demonstrated in how particular discourses are used or not used; which language is used to represent and interpret events and experiences. Blackler and McDonald ${ }^{19}$ found that when people are working in interorganizational knots that they are no longer bounded by the knowledge, practices, and relationships that regulate their work, that they have to invent new practices and orderings of work and manage continually fluctuating power relations.

New practices may include communicating more clearly about expectations and ways of doing things. In research on knots occurring in academic research teams, Laitinen et al., ${ }^{20}$ revealed that practices on storing, coding and describing data varied from researcher to researcher impacting the ability of the overall team to find solutions to data management problems. These researchers had to develop a code book particular to this research. Eppich et al. ${ }^{21}$ more specific to medical teams found that medical team members had to speak up and even assign critical language for calling a time out when they had patient safety concerns specific to their area of expertise because of misunderstandings between those team members. Similarly, Andreatta et al. ${ }^{22}$ found that often there were discrepancies between clinical practice and institutional policies in interdisciplinary medical team influencing how members saw and responded to emergencies. These discrepancies had to be recognized and resolved in order for team members to respond similarly and consistently.

When the RRT comes together, knots are created to find solutions to specific problems and are improvised. As this occurs, boundary lines are crossed, multiple roles are negotiated all at once, and power structures are dealt with that are inherent in medical care. Knot negotiations such as these can influence whether someone speaks up, criticizes other team members, or keeps silent.

To better understand how RRTs function and communicate and ultimately improve RRT effectiveness, the purpose of this study, using activity theory and knotworking as a framework, is to help explicate how professional members of an obstetrical RRT team assert agency in knots and manage power structures.

RQ1: What is the nature of knots that arise for RRT teams?

RQ2: How is the knot negotiated by multiple roles in an $R R T$ ?

RQ3: How is power demonstrated as people interpret and represent their roles in the knot?

\section{Materials and Methods}

\section{Organizational setting overview}

A research team, including myself, conducted this study with the labor and delivery unit of an urban teaching 
university hospital in the Western United States that serves childbearing women of diverse medical and social needs. Maternity care is hospital service-based and also includes private practice and midwifery. The hospital has 476 inpatient beds and 52 Newborn Intensive Care Unit beds. Labor and Delivery has 14 inpatient beds and three Operating Rooms. Obstetrical Emergency Services has nine beds. In 2013 the hospital had 3,859 deliveries.

An OB Rapid Response Team (OBRR) process was implemented in 2008. A number of people across the roles of the OBRR (chief OB resident, Jr. OB resident, OB attending, OB anesthesiologist, L\&D charge nurse, $1^{\text {st }}$ responder $(\mathrm{RN}), 2^{\text {nd }}$ responder (RN), backup (RN) and scrub (MA)) complained about the chaos that was involved any time the OBRR occurred. Complaints included who should make the call, what roles should be performed, and questions about who should be responsible for assigning roles. Complicating matters is personnel turnover. While the stakeholder role remains consistent, the stakeholders themselves do not. The OBRR is implemented when someone calls the hospital dispatcher who sends out a page to the team. When the call is made, the stakeholders all have specific tasks that vary with the specific emergency.

The impetus of this study occurred when one of the nurses involved in quality improvement wanted advice on how to make the process sustainable. After obtaining Institutional Review Board approval, a qualitative approach was used to highlight the voices and views of participants. Qualitative research methods are suited for initial explorations of complex phenomena and are often employed as groundwork for theory formation or experimental work. ${ }^{22}$ In this study, rather than beginning with theoretically guided questions, qualitative analysis allowed for an examination of ERR's from insiders' perspectives allowing their descriptions and analyses of the context to emerge. Rundell, Devers, and Sofaer suggest qualitative research is especially helpful in health care settings in bridging the gap between quantitative studies of managed care and media accounts because it helps explain what forms of managed care implemented in various ways might influence provider behavior under certain conditions or circumstances in ways that might improve or harm quality of care. $^{23,24}$

Nine focus groups occurred, held over a two-week period for each of the stakeholders involved in OBRR teams. Stakeholders included Obstetrical Residents (OB RES), Obstetrical Attending (OB ATT), Obstetrical Anesthesiologists (OB ANES), other attending physicians (ATT), Advanced Practice Nurses (APRN), Charge Registered Nurses (CRN), Staff Nurses (SRN), Midwives, and Medical Assistant/Health Unit Coordinators (MA/HUC). Though these groups are multidisciplinary, focus groups were held with individual stakeholder groups for two reasons: first to alleviate power issues such as the possibility of medical assistants being intimidated by physicians, secondly, to develop an understanding of how each stakeholder group viewed the rest of the team from their own perspective. The goal of the focus groups was to understand the perceived barriers involved in the OBRR. Each of the focus groups was approximately 60 minutes long and participants from each group/role were asked to voluntarily participate. Researchers asked participants open-ended questions in order to help them describe their experiences and responses to working in an OBRR team. Responses were recorded by research assistants on large posterboards situated around the room.

Results from the focus groups demonstrate that knots occur, are negotiated, and that power struggles within the knot occur. These are described in the following section.

\section{Coding}

In order to understand the process, activity theory and more specifically knotworking was used as a framework. Though the focus of knotworking in the past has been primarily in educational settings, such examination of pressure and pulsations seems to help explain interconnections and subtleties of crisis teamwork in new ways.

For this project, I used qualitative methods of analysis to answer the research questions. This methodological approach embraces a humanistic orientation to understanding and representing participants' realities. ${ }^{25,26}$ Focus group comments $(n=709)$ were transcribed verbatim and then analyzed. To provide confidentiality, participants are identified only by role. ${ }^{27}$ In order to stay true to the raw materials, qualitative content analysis was used to code focus group comments. ${ }^{28}$ This methodology allows for attention to content or contextual meaning of the text. ${ }^{29}$ Each comment was identified by role and assigned an attribute after asking What is happening here and What are the participants main concerns? ${ }^{30}$ Attributes were such things as workflow, problem, success, transition. Attributes were then examined for language which would identify specific agentic actions. Finally, the assigned attributes and actions were discussed by all involved in the project to determine if they rang true, what they meant, and whether these could be applied consistently. Agentic actions were used to guide the reporting and discussion of qualitative data in order to present the focus group findings. Two hundred sixteen comments were not coded as they were listed in focus group notes as such things as OB Team or SBAR (an evaluation tool), but did not carry valence or clarifying information.

Of Engestrom's five agentic actions, steps four and five were not coded from focus groups because these comments were centered around things that had happened in the past. It was not until after the focus group results were compiled and shared with stakeholders that changes began to occur. Steps four and five are more directed at future actions. These steps emerged through later discussion with the OBRR team and were shared with the research team. 


\section{Results}

Research question one asked about the nature of knots. This question was answered by examining all comments for thematic content to determine what and where pulsations and negotiations (knots) occurred. Results are presented using comments. However, though this is a qualitative study, numbers and percentages are also included to demonstrate how often comments were made and how big of a percentage they represent in comparison to other themes.

Four themes emerged from 485 overall comments: process $(\mathrm{n}=309,63.71 \%)$, communication $(\mathrm{n}=75$, $15.46 \%)$, role $(\mathrm{n}=73,15.05 \%)$ and training $(\mathrm{n}=28,5.77 \%)$ (Figure 1).

Process had the highest number of comments. Process comments referred to anything that had to do with the way the OBRR functioned. In examining each stakeholder, process has the highest percentage of comments across all four themes except for midwives. Comments included such things as The charge nurse stands in the corner...the doctor is not directing activity in the room (SRN) and the senior anesthesia resident cannot put [the] patient to sleep without an OB ATTing in the room (OB RES). All the stakeholders focused on process; however, $88 \%$ of all comments made by OB RESs were process comments while only $44 \%$ of the midwives total comments were process related.
Comments about communication revolved around the way stakeholders transferred information before, during, and after the OBRR was called. Often stakeholders asked for clarification, translation, or discussed differences in articulation as varying stakeholders as well as individual personalities negotiated the OBRR. One MA said: The $O B$ Provider should announce when it's a shoulder dystocia so we can get call the OB Rapid Response and get the help we need quicker. Midwives were concerned about communication as they negotiated the complexities of coming into onsite situations from the outside where OB RESs and RNs were already working with patients and power was not always transparent. One RN wondered How often do you communicate with new versus experienced nurses [about patient care]? Midwives also felt they (the midwives) were much more conscientious about discussing patient treatment with the patient than other roles.

Communication issues focused on discursive differences between groups such as nurses and MA/HUCs feeling like they could not call an OBRR, and physicians feeling like these calls were not always clear because not all OBRRs called were emergencies that needed their involvement. Midwives were the most focused on communication with $44 \%$ of their comments relating to communication. At the opposite end, CRN's comments were least focused on communication - only $8.2 \%$ and most of these were more about how the process was being communicated than about how people were inter-

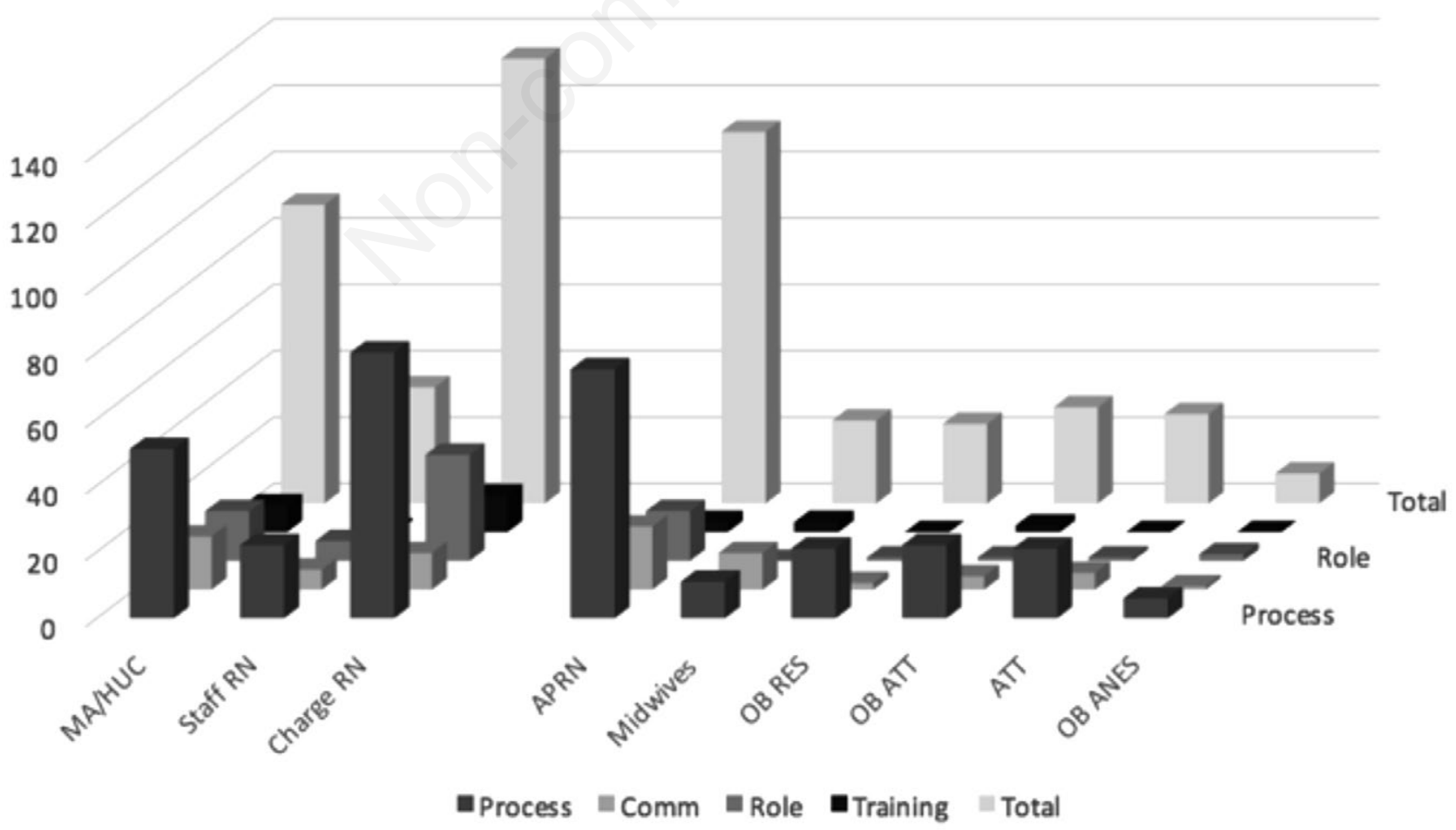

Figure 1. Comments, by roles and themes 
acting: We need more clarity about who gets paged (CRN).

Seventy-three comments centered on the role played in the OBRR team. When examining the total number of role comments, the stakeholders with the highest percentage of role comments $(23.88 \%)$ were CRNs. This relates to their role as the person who assigns various stakeholders to the team on each shift. One CRN asked, Who checks whether the cart is unlocked and stocked - the MA/HUC?

Another said:

When I was first on the team and was assigned to be the $2^{\text {nd }}$ responder in an emergency Cesarean Section, I really didn't know what I was supposed to do so I would just start to do the $1^{\text {st }}$ responder role because I knew those tasks.

An APRN suggested, [We need] clear expectations of everybody's role. Other role comments focused on how the OBRR was working together: It's not my patient but our patient (APRN) and explaining those interconnections to patients We need to educate on the hand-off and explain roles to patients (SRN). CRNs and Midwives did not make any comments about role.

The fourth theme centered on issues about training. Twelve percent of the midwife's comments were about training: Residents need training on working with midwives - get on agenda/need to change culture/shift attitudes (Midwife). Eight percent of CRNs comments were about training. These were mainly about the need for training and education [We need] to improve training and have better education, better orientation, and review roles (CRN). One RN described the following:

When the emergency light goes off and lots of people run into the room there can be a lot of noise and commotion. Sometimes this seems to make the situation worse because staff are unsure of what role they are in and what they should be doing in that role or staff who are not part of the team run in to try to help and then there are too many people in the room.

Another RN noted:

I had a shoulder dystocia and pushed the emergency light. Some newer staff nurses came running into help. The Charge Nurse was in a different delivery and didn't hear the page. The baby was born floppy with minimal respiratory effort. Because these newer nurses didn't have much experience with resuscitating babies, Ineeded to do this until the Pediatric team arrived.

Eight percent of MA/HUCs comments were about training as well but these were mainly about the need for their own training. [We] need more training on OBRR (MA/HUC). The only physician stakeholder group with any comment about training was the OB attending physicians who are in charge of training interns and residents. MA/HUCs made more comments about training (or need for training) than any other role. There were three roles who did not make any comments at all about training: OB RES, ATT, and OB ANES.

\section{Knot negotiation}

The second research question asks how the knot is negotiated by multiple roles in an RRT. This question was answered by coding focus group comments for Engestrom's ${ }^{18}$ five participant agentic actions. These actions include: i) Resisting the interventionist or management; ii) Explicating new possibilities or potential in the activity; iii) Envisioning new patterns or models of the activity; iv) Committing to concrete actions aimed at changing the activity and expressing this through commissive speech acts; v) Taking consequential actions to change the activity. Actions one through three were examined through focus group comments. Actions four and five will be discussed later in the paper.

\section{Resistance}

Resistance, or pushing back against the interventionist or management, took place as team members expressed unhappiness with the current status. Discursive work was evident in comments that focused on resistance as evidenced by these comments: Doctors [are] trying to dictate - telling the nurses not to follow protocol (SRN) and In the Cesarean Sections, the charge nurse is not always active in response ( $S R N)$. One $\mathrm{CRN}$ said:

I have been told by some of the doctors to not push the emergency light because they feel that they have all the staff they need in the room to handle the situation. At times I don't think we need to call the $O B$ Rapid Response because all team members are in the room. But sometimes this isn't true and we end up having to wait for the OB Attending or the Anesthesia Attending.

Sometimes there was resistance in assignments given.

The Charge Nurses assign the roles at the beginning of the shift but sometimes there isn't enough experienced staff and newer staff are assigned and that can be a problem if they have never been in that role before $(S R N)$.

APRN's asked the most questions, OB ANES did not ask any.

\section{Explicating}

Explicating involves explaining and looking for potential in the activity. Explicating comments focused on defining or explaining the OBRR process. These included such things as: Midwives typically talk through the process to avoid the RR button from being pushed during shoulder dystocias (Midwife), and the clarity of communication depends on the team (who is on it at any given time) (CRN). One SRN even said, Some of the OB Providers should speak louder so that we can all hear the plan. CRNs had the highest percentage of comments that explicated something about the OBRR process. At the same time, OB ANES did not make any explicating comments. 


\section{Envisioning}

Envisioning involves looking for new patterns or models. Comments in this category were about envisioning changes for the future or looking for solutions. One comment was a suggestion that it would be helpful if team members' names from the incoming shift should be written next to the previous member's names on the white board (OB RES). Another was that including pictures of team members on the white board would be helpful (OB ATT). SRNs and OB RESs made few comments in this category.

After focus group results were shared with the OBRR stakeholders, steps four and five emerged. Step four, committing to concrete actions aimed at changing the activity and expressing this through commissive speech acts, occurred in a number of ways. As focus group comments were shared, stakeholders started discussing the points they thought were most pertinent and valuable and where changes could be implemented. The changes were then taken back to the larger stakeholder groups and discussed. For example, the OB ANES saw value in having a second responder dedicated to them, and recognized that many of the staff were unsure of how to perform the tasks expected of them, and that there was a need for increased and more specific training.

Step five, taking consequential actions to change the activity, occurred later as well. Some of the specific changes include i) Agreement by all stakeholders that anyone can push the come now button for OBR; ii) Assignment of team members prior to shift change; iii) Safety briefing of team members; iv) Creation and distribution of a document by midwives that describes the various stages of consultation/co-management/hand-off; v) change of the response call for postpartum haemorrhage to include Stage 1 and Stage 2; vi) creation of a concept called perfect practice which means a standardized protocol is followed.

\section{Power}

Research question three asked how power and power imbalance may influence the team process. Power is inherent within a medical setting. Physicians' words and actions carry more weight than those of an intern or resident. Often an intern or resident exhibits more power than a nurse, even those with many more years of experience. Medical assistants typically have the least amount of power. These power imbalances were apparent in the OBRR and the way discourse and negotiation occurred. One example of power imbalance was evident when nurses felt that interns, residents, and other physicians were chastising them. However, interns and residents said they never chastised: We don 't raise our voices or question them. We don't know why the nurses say we do.

Power is even more evident when looking at types of comments by role. All roles made the most overall comments about process but those with power to change train- ing had higher percentages of comments in this category: Midwives (12\%), CRNs (8.2\%), and OB ATTs (6.89\%). The person with the most ability to change roles within the OBRR is the CRN. One MA said:

The rule is that staff should be here at least 6 months before they can be on the RR team but sometimes CRN assign a newer MA to be the scrub and they are a little freaked out by that.

Power issues also arose when looking at agentic actions. Those with less power spent more time questioning and explicating. Of particular note was the repeated dissonance those in low power felt interacting with higherpowered/ranked individuals such as the OBs. One term the low-power participants used repeatedly was unappreciated, indicating that for them, one of the major indicators of the success of the OBRR was their contribution to making everything flow smoothly. Those with more power focused on envisioning. In fact, OB ANES ONLY envisioned.

Alternatively, it may be that the MA/HUCs and CRNs had the most concerns about roles because their specific role in the OBRR team deals with the greatest number of individuals and processes in the knot. In other words, though this may be a power issue, it may be also because they observed and interacted with a larger proportion of the threads in the knot than the OBs, thus commenting on those roles the most frequently.

Ultimately, process spans boundaries, communication, role, and training which all emerge more specifically with stakeholder role and power. Research question one asked about the nature of the knots implicated in the OBRR teams. This research identified four areas of pulsations or knots: process, communication, role, and training. Research question two asked how the knot is negotiated by roles. All five of the agentic actions were demonstrated. However, the first three occurred during the focus groups - question, explicate, and envision. The three actions occurred and different roles were more dominant in different actions. The other two action steps (committing and taking action) occurred in real time as the team handled crises and worked through the questioning, explicating, and envisioning process. Research question three asked how power is demonstrated as people interpret and represent their roles in the knot. Results provide similar insight into the complicated process of power imbalance in medical situations.

\section{Discussion}

As stakeholders develop procedures to negotiate the knots that occur when an OBRR is called, each person performs within boundaries of roles and with their own agency and motivation for that performance. This fluid interactivity creates shifting discursive patterns and knots that could be mapped during focus groups with stakeholders. Patterns emerged such as those lower in power focus- 
ing more on questioning and explicating and those with more power focusing on envisioning. This mapping shows how examining individuals, objectives and tools is not enough. Community membership, labor division, and rules for interaction (Engestrom, 2000) also must be included to understand how OBRR teams work together.

Process comments illustrate the importance of analyzing patterns that emerge as people talk about the OBRR process. Process was by far the most important aspect of completing a successful OBRR. Analysis of patterns shows that the majority of the discourse and problems that occurred were about the process itself. Mapping the comments shows OB RESs having the most process comments with twice the number of comments as midwives. This could be because midwives are in and out of the hospital and not as involved in day-to-day procedures while $\mathrm{OB}$ RESs are not only there but are required to report to their supervisors on activities. Such mapping shows how fluid the process is and how the discursive work focuses on different aspects of the same task as they locate themselves and engage with others. Examining process through the lens of knotworking helps to explain where complications might occur, how to anticipate issues that might arise, how actions are demonstrated and at which point.

In this OBRR, the discursive practices demonstrate how complex relationships and knots are in crisis teams - nurses were overruled by interns with less experience, doctors gave instructions but were perceived as yelling at nurses or MA/HUCs insisted they needed more training on OBRR roles and expectations. The complexity includes the crisis being managed and also how process, communication, role and training influence what actually happens.

As different stakeholders interact, tension occurs as stakeholder roles are negotiated. One example of this was when Midwives felt Residents did not understand how to work with the Midwives. Each role had its own responsibilities and all stakeholders were committed to the wellbeing of the patient but how that interaction occurred was sometimes messy and complicated and misunderstood.

In addition to the culture is the fact that the composition of the team is virtually never the same creating different knowledges, capacities, and configurations, which emerge in discursive work as individuals interact in the OBRR. The actions and stories of each at least partly construct the responses of the other stakeholders such as when stories told by Midwives emphasized how they communicated with the patients and MA/HUCs felt Midwives took too long to call the RR. Additionally, because of the fluid nature of the knot some forms of participation may resist fixation and invite more frequent re-negotiation than other types of RRTs. This is especially true of Interns and the frequency with which they change. Because of this, the training component becomes critical in order to explicitly signal the discursive work they are doing by promoting and modeling critical attunement to language and negotiation between overlapping discursive communities and how to span boundaries.

Further complicating the productive activity of caring for patients, are the tightening and loosening of tensions or knots within the OBRR. Knot pulsations occurred when different stakeholders had different definitions about what the come now call for OBRR meant. Different power levels affected definitions as well. When a doctor said come now everyone came. When an MA/HUC said come now it was not always seen as an emergency. SRNs sometimes called an OBRR and physicians then yelled at them later because the physician did not feel the call was warranted. Translating those terms and procedures into common language was an important step that occurred after focus groups were over, problem areas had been identified, and pinch points identified. Understanding knotworking actions can help improve the process of OBRR by identifying particular places that knots may occur.

While Engestrom's ${ }^{18}$ five participant agentic actions are evident in the OBRR team, this study demonstrates that the five actions occur but they may in occur in stages. Focus group comments exhibit the actions of resisting the interventionist or management through questioning statements of frustration. The second action, explicating new possibilities or potential in the activity, is evident in the stakeholder role of CCRNs as they explained what was going on as OBRR teams worked together and looking at the process in varied ways. The third action, envisioning new patterns or models of the activity was more apparent in the stakeholder roles of physicians. The comments identify new areas for improving the team. Such comments indicate the possibility of making changes possible whereas those with less power, even when envisioning, are looking towards those with more power to actually make the changes possible.

The first three actions are manifest in focus groups; however, the last two only became evident later on as the first three actions were analyzed for ways to improve the OBRR. Prior to this point, stakeholders voiced opinions separately but not collectively. The collaborative point occurred when discussions took place based on the focus groups, specific changes were recognized and agreement was reached on what changes to implement. Step five, taking consequential actions to change the activity, occurred after changes were agreed upon by stakeholder groups and the changes continued to happen. Actions were implemented carefully and thoughtfully to help make the OBRR team more effective both in patient care and in communication among stakeholder roles and stakeholder satisfaction. The findings of this study indicate that RRTs can get better at understanding and training.

Resistance, explicating, envisioning, committing to action, and changing the activity were demonstrated both in the focus groups and in follow-up activity. The stakeholders struggled to i) make sense of what happens in the knots; ii) improvise collaborative practices; iii) negotiate 
colliding knowledge systems and interests of multiple identities and roles. Some of the resisting helped later negotiations by aiding in understanding what was happening. For example, OB Residents voiced opinions about the value of the OBRR; however, they did not want the chaos that happens when the actual OBRR is called. Understanding what is seen as value and what is seen as chaos helps to develop action plans for changing the activity in the future. Viewing OBRR teams as knots helps to reconceptualize possibilities for training and procedures for other types of emergency response teams. This finding supports the fact that the actors in the OBRR found knotworking and its implications for understanding the process as useful.

Leach and $\mathrm{Mayo}^{3}$ bemoan the lack of process research in crisis teams. This study demonstrates the importance of such study. Process issues are inherent in every aspect of the OBRR. Process, communication, role and training are all themes which emerge from the focus group comments. Power struggles occur at varying levels within the knot and need to be negotiated over and over. Blackler and $\mathrm{McDonald}^{19}$ (p. 840) identify power in knots as both an ongoing product and medium for collaborative activity. They go on to suggest that studying power in knots helps identify what is normal, and how new configurations and disruptions are negotiated.

This study validates the need for negotiating power relationships in order for RR groups to succeed. In our research, those with power over time quit talking about issues: they do not need to discuss power because they already have power. Lower power stakeholders do not speak because they do not see that they have power. For example, one of the researchers suggested to the nurses that they speak up. The researcher's claim that the nurses have power is possibly correct. However, telling the nurses to speak up is not useful if they do not believe they can. Power imbalances are normal and inherent in medical settings.

In OBRR normal channels of workflow are disrupted, and stakeholders are expected (even encouraged) to work at a high level of autonomy and to support the team effort. One of the issues with a RR process is the move away from the routine interaction patterns in a hospital setting. This is when the hierarchy of the hospital culture becomes an impediment such as when OB residents may have limited experience as compared to many of the nurses; however, in the current hierarchical hospital culture, they are considered more authoritative.

\section{Conclusions}

When viewed through the lens of Activity and knotworking theory, RRTs become more understandable. The dominant concern of RRTs - process, or having a successful outcome as a result of what happens during the RRT indicates that knotworking's ability to analyze and under- stand roles and processes within RRTs could be critical to improving the quality of such RRTs. Taking individual elements such as stakeholders or divisions of labor yields a partial picture, but does not demonstrate the pulsing, everchanging aspect of teams, which come together with specific roles and stakeholders. A single-minded focus on training for system errors may miss important pulsations beyond written and practiced protocol such as how and when the OBRR is being called. Such pulsations may include a nurse wanting to push the button but not wanting to get yelled at, or being comfortable with the expectation that they need to play a more active role in monitoring the tone and flow of the team during an emergency instead of handing off to a physician.

The application of Activity and knotworking theory to the healthcare environment is promising because it helps providers move beyond just a basic understanding of teamwork to an understanding of the motivations that occur within that teamwork, how stakeholders enact resistance and explain the situation, and ultimately how they commit to change and then enact those changes. Such a move is necessary to go beyond tracking and reporting outcomes to understand why sometimes routine interaction patterns do not work and why some stakeholders do not adapt well to varying circumstances. With this understanding, training programs and interventions could occur which would help providers reconfigure RRTs, train, and increase knowledge sharing to reduce patient harm.

The use of focus groups was helpful in developing understanding of what happens in teams, however it was also a limitation. This was an exploratory study and was limited to people's perceptions without observations to substantiate those perceptions.

While knotworking and activity theory have obvious benefit in understanding - and by extension, improving group dynamics in the RRT setting, researchers and leaders can apply these principles in many more critical settings, such as law enforcement task forces, natural disaster relief efforts, search and rescue operations, or other types of emergency responses. Often leaders of RRTs focus on step-by-step training but not on where pulsations and knots occur. Such teams are complicated, involving multiple roles and ever-changing personnel. An understanding of knotworking can help RRT teams understand where knots might occur and anticipate not only problems that are already occurring but how even envisioning future events can create misunderstanding and knots.

Future research needs to focus on how each aspect of the knot can be examined to determine if there are particular times or emergencies that certain types of knots occur and with which stakeholders. Actual observations could help to validate or refute the types of recollections that occurred in these focus groups. A follow-up, longitudinal study could also determine if raising awareness of the fluidity of roles and power makes a difference in practice over time. 


\section{References}

1. Yu T, Sengul M, Lester RH. Misery love company: the spread of negative impacts resulting from an organizational crisis. Acad Manag Rev 2008;33:452-72.

2. Stachowski AA, Kaplan SA. The benefits of flexible team interaction during crises. J Appl Psychol 2009;94:1536-43.

3. Leach LS, Mayo AM. Rapid response teams: qualitative analysis of their effectiveness. Am J Crit Care 2013;22:198210.

4. Laxmisan A, Hakimzada F, Sayan OR, et al. The multitasking clinician: decision-making and cognitive demand during and after team handoffs in emergency care. Int J Med Inform 2007;76:801-11.

5. Lingard L, Espin S, Evans C, Hawryluck L. The rules of the game: interprofessional collaboration on the intensive care unit team. Crit Care 2004;8:R403-8.

6. Shearer B, Marshall S, Buist MD, et al. What stops hospital clinical staff from following protocols? An analysis of the incidence and factors behind the failure of bedside clinical staff to activate the rapid response system in a multi-campus Australian metropolitan healthcare service. BMJ Qual Saf 2012;21:569-75.

7. Rapid response teams: a systematic review and meta-analysis. Retrieved October 10, 2014. Available from: http://archinte.jamanetwork.com/

8. Janss R, Rispens S, Segers M, Jehn KA. What is happening under the surface? Power, conflict and the performance of medical teams. Med Educ 2012;46:838-49.

9. Varpio L, Hall P, Lingard L, Schryer CF. Interprofessional communication and medical error: a reframing of research questions and approaches. Acad Med 2008;83:576-81.

10. Engestrom Y. Activity theory as a framework for analyzing and redesigning work. Ergonomics 2000;43:960-75.

11. Lundby K. Interdisciplinarity and infrastructure: mediation and knotworking in communication research. NORDICOM Rev 2007;28:195-209.

12. Kuutti K. Activity theory as a potential framework for human computer interaction research. In: Nardi BA, ed. Context and consciousness: activity theory and human-computer interaction. Cambridge, MA: MIT Press; 1996. pp 17-24.

13. Engestrom Y. Learning by expanding: an activity-theoretical approach to developmental research. Helsinki: Orienta-Konsultit; 1987.
14. Engestrom Y. Communication, discourse and activity. Commun Rev 1999;3:165.

15. Engestrom R. Voice as communicative action. Mind Cult Activ 1996;2:192-215.

16. Engestrom Y, Engestrom R, Vahaaho T. When the center does not hold: the importance of knotworking. In: Chaiklin S, Hedegaard M, Jensen UI, eds. Activity theory and social practice: cultural-historical approaches. Aarhus: Aarhus University Press; 1999. pp 345-374.

17. Engestrom Y. New forms of learning in co-configuration work. J Workplace Learn 2004;16:11-21.

18. Engestrom Y. From design experiments to formative interventions. Theory Psychol 2011;21:598-628.

19. Blackler F, McDonald S. Power, mastery and organizational learning. (Organizational learning: past, present and future). J Manag Stud 2000:832.

20. Engeström Y, Kaatrakoski H, Kaiponen P, et al. Knotworking in academic libraries: two case studies from the University of Helsinki. Liber Quarte: J Eur Res Libr 2012;21:387-405.

21. Eppich WJ, Brannen M, Hunt EA. Team training: implications for emergency and critical care pediatrics. Emerg Crit Care Med 2008;20:255-60.

22. Andreatta P, Frankel J, Smith SB, et al. Interdisciplinary team training identifies discrepancies in institutional policies and practices. Am J Obstet Gynecol 2011;205:298.

23. Sofaer S. Qualitative methods: what are they and why use them? Health Serv Res 1999;34:1101-18.

24. Rundall TG, Devers KJ, Sofaer S. Qualitative methods in health services research: introduction. Health Serv Res 1999;34:1091-9.

25. Lindlof TR, Taylor BC. Qualitative communication research methods. 2nd ed. Thousand Oaks, CA: Sage; 2002.

26. Tracy S. Qualitative research methods. Malden, MA: WileyBlackwell; 2013.

27. Lapan SD, Quartaroli MT, Riemer FJ. Qualitative research: an introduction to methods and designs. San Francisco: Wiley/Jossey-Bass; (In press).

28. Hsieh H-F, Shannon SE. Three approaches to qualitative content analysis. Qual Health Res 2005;15:1277-88.

29. Tesch R. Qualitative research: analysis types and software tools. Bristol, PA: Falmer; 1990.

30. Thornberg R, Charmaz K. Grounded theory. In: Lapan SD, Quartaroli MT, Riemer FJ, eds. Qualitative research: an introduction to methods and designs. San Francisco, CA: Jossey-Bass; 2012. pp 42-67. 